\title{
Tangeretin Improves Glucose Uptake in a Coculture of Hypertrophic Adipocytes and Macrophages by Attenuating Inflammatory Changes
}

\author{
Hye-Sun Shin ${ }^{1}$, Seong-Il Kang ${ }^{1}$, Hee-Chul Ko ${ }^{2}$, Deok-bae Park ${ }^{3}$ and ${ }^{\dagger}$ Se-Jae Kim ${ }^{1}$ \\ ${ }^{1}$ Department of Biology, Jeju National University, Jeju Special Self-Governing Province, Korea \\ ${ }^{2}$ Jeju Sasa Industry Development Agency, Jeju National University, Jeju Special Self-Governing Province, Korea \\ ${ }^{3}$ Department of Medicine, Jeju National University, Jeju Special Self-Governing Province, Korea
}

\begin{abstract}
Obesity is characterized by a state of chronic low-grade inflammation and insulin resistance, which are aggravated by the interaction between hypertrophic adipocytes and macrophages. In this study, we investigated the effects of tangeretin on inflammatory changes and glucose uptake in a coculture of hypertrophic adipocytes and macrophages. Tangeretin decreased nitric oxide production and the expression of interleukin (IL)-6, IL-1 $\beta$, tumor necrosis factor- $\alpha$, inducible nitric oxide synthase, and cyclooxygenase-2 in a coculture of 3T3-L1 adipocytes and RAW 264.7 cells. Tangeretin also increased glucose uptake in the coculture system, but did not affect the phosphorylation of insulin receptor substrate (IRS) and Akt. These results suggest that tangeretin improves insulin resistance by attenuating obesity-induced inflammation in adipose tissue.
\end{abstract}

Key words : Tangeretin, Adipocytes, Macrophages, Inflammation, Glucose uptake

\section{INTRODUCTION}

At the cellular level, obesity is caused by an increase in the number and size of adipocytes in adipose tissue (Spiegelman and Flier, 1996). Adipocytes accumulate excessive concentrations of triglycerides; this interferes with the signaling pathways involved in the normal metabolism of adipocytes, causes insulin resistance, and may even lead to metabolic syndrome abnormalities (Kahn and Flier, 2000). Obesity may be further characterized as a state of chronic low-grade inflammation marked by the infiltration of macrophages into adipose tissue (Weisberg et al., 2003; Xu et al., 2003). Interactions between hypertrophic adipocytes and infiltrated macrophages aggravate inflammation and lead to insulin resistance in obese adipose tissue (Dandona et al., 2004; Fernández-Real and Ricart, 2003).

Adipose tissue macrophages are a major source of proinflammatory cytokines, which play important roles in chronic inflammatory responses in obese adipose tissue (Itoh et al., 2011). For example, tumor necrosis factor (TNF)- $\alpha$ directly decreases insulin sensitivity and increases lipolysis in adipocytes (Hotamisligil et al., 1995; Zhang et al., 2002). Interleukin (IL)-6 leads to hypertriglyceridemia in vivo by stimulating lipolysis and hepatic triglyceride secretion (Nonogaki et al., 1995). In addition, induced nitric oxide synthase (iNOS) and TNF- $\alpha$ are required for the development of obesity-induced insulin resistance in mice (Perreault and Marette, 2001). The pro-inflammatory cyto-

\footnotetext{
Manuscript received March 8, 2017, Received in revised form March 10, 2017, Accepted March 12, 2017

${ }^{\dagger}$ Corresponding Author : Se-Jae Kim, Department of Biology, Jeju National University, Jeju Special Self-Governing Province, Korea. Tel : +82-64-7543529, Fax : +82-64-756-3541, E-mail: sjkim@jejunu.ac.kr

This is an Open Access article distributed under the terms of the Creative Commons Attribution Non-Commercial License (http:// creativecommons.org/licenses/by-nc/3.0) which permits unrestricted non-commercial use, distribution, and reproduction in any medium, provided the original work is properly cited.
} 
kines produced by both adipocytes and recruited macrophages might synergistically decrease insulin sensitivity (Dandona et al., 2004; Hotamisligil et al., 1995).

Tangeretin, a type of polymethoxyflavone (PMF), is found in citrus plants and exerts anti-inflammatory, anticancer, and anti-obesity effects (Chen et al., 2007; Choi et al., 2007; Kim et al., 2012; Morley et al., 2007). It was used in traditional medicine for treatment of various diseases. Also, tangeretin modulates glucose metabolism in streptozotocin induced diabetic rats (Sundaram et al., 2014). However, little is known about the effects of tangeretin on the inflammatory responses and insulin resistance caused by direct interactions between adipocytes and macrophages. In the present study, we investigated the effects of tangeretin on inflammatory changes and glucose uptake in a coculture of hypertrophic adipocytes and macrophages.

\section{MATERIALS AND METHODS}

\section{Cell culture}

We obtained 3T3-L1 preadipocyte cells and RAW 264.7 murine macrophage cells from the American Type Culture Collection (Rockville, MD, USA) and the Korea Cell Line Bank (Seoul, South Korea), respectively. The cells were cultured in Dulbecco's modified Eagle's medium (DMEM; Gibco BRL, Gaithersburg, MD, USA), which contained 1 $\%$ penicillin/streptomycin (PS) (100 units penicillin/mL and $100 \mathrm{pg}$ streptomycin $/ \mathrm{mL}$ ) and was supplemented with either $10 \%$ bovine calf serum (BCS, Gibco, NY, USA) or $10 \%$ fetal bovine serum (FBS, Gibco, NY, USA), at $37^{\circ} \mathrm{C}$ in a $5 \% \mathrm{CO}_{2}$ atmosphere. To prepare hypertrophic adipocytes, post-confluent preadipocytes (designated as day 0) were cultured until confluent in differentiation medium [DMEM containing 1\% PS, 10\% FBS, $0.5 \mathrm{mM}$ 3-isobutyl1-methylxanthine (IBMX), $1 \mu \mathrm{M}$ dexamethasone, and 1 $\mu \mathrm{g} / \mathrm{mL}$ insulin] for 2 days. The cells were then cultured for 2 more days in DMEM containing 1\% PS, 10\% FBS, and
$1 \mu \mathrm{g} / \mathrm{mL}$ insulin. Thereafter, the hypertrophic 3T3-L1 cells with larger lipid droplets were prepared in post-differentiation medium (DMEM containing 1\% PS and 10\% FBS) for 14 days, which was replaced every 2 days. Co-culturing of adipocytes and macrophages was performed in accordance with a previously described protocol (Jeon et al., 2015; Shin et al., 2016). RAW 264.7 macrophages were plated in culture dishes containing serum-starved DMEM. Then, they were plated onto a hypertrophic 3T3L1 monolayer and the cocultured cells were incubated in serum-starved DMEM for $24 \mathrm{~h}$. Equal numbers of RAW 264.7 macrophages and 3T3-L1 adipocytes were cultured separately as controls. After 24 h, tangeretin (Wako Pure Chemical Industries, Osaka, Japan) was added to the cocultured cells at various concentrations for an additional 24 h. The supernatants and cells were collected and stored at $-20{ }^{\circ} \mathrm{C}$ until use. Cell cytotoxicity was determined by lactate dehydrogenase $(\mathrm{LDH})$ release assay (Takara Shuzo Co., Otsu, Japan).

\section{Measurement of nitrite release}

The amount of nitrite was determined by colorimetric assay. Briefly, $100 \mu \mathrm{L}$ of cell culture medium was mixed with an equal volume of Griess reagent ( $1 \%$ sulfanilamide and $0.1 \%$ naphthylethylenediamine in $5 \%$ phosphoric acid) and incubated at room temperature for $10 \mathrm{~min}$. The absorbance at $540 \mathrm{~nm}$ was recorded using a microplate reader (BioTek instrument Inc., Winooski, VT, USA). The nitrite concentration was determined by extrapolation from a sodium nitrite standard curve.

\section{Western blot analysis}

Cells were homogenized with lysis buffer $[1 \times$ RIPA (Upstate Cell Signaling Solutions, Lake Placid, NY, USA), 1 $\mathrm{mM}$ phenylmethylsulfonyl fluoride, $1 \mathrm{mM} \mathrm{Na}_{3} \mathrm{VO}_{4}, 1 \mathrm{mM}$ $\mathrm{NaF}, 1 \mu \mathrm{g} / \mathrm{mL}$ aprotinin, $1 \mu \mathrm{g} / \mathrm{mL}$ pepstatin, and $1 \mu \mathrm{g} / \mathrm{mL}$ 
leupeptin] and incubated on ice for $1 \mathrm{~h}$. Cell lysates were subjected to sodium dodecyl sulfate polyacrylamide gel electrophoresis (SDS-PAGE) and transferred to polyvinylidene difluoride membranes. These membranes were then blocked with a solution of $0.05 \%$ Tween $20 /$ Tris-buffered saline containing 5\% non-fat dry milk for $1 \mathrm{~h}$ at room temperature. Antibody against iNOS was obtained from Calbiochem (San Diego, CA, USA), and antibody against COX2 was obtained from BD (Mountain View, CA, USA). Anti-phospho-AKT and anti-insulin receptor substrate (IRS) antibodies were obtained from Santa Cruz Biotechnology (Santa Cruz, CA, USA). Antibodies against phospho-IRS and AKT were obtained from Cell Signaling Technologies (Beverly, MA, USA). After incubation overnight at $4{ }^{\circ} \mathrm{C}$ with the indicated primary antibody, the membranes were incubated with the horseradish peroxidase-conjugated secondary antibody (Jackson ImmunoResearch, West Grove, PA, USA) for $1 \mathrm{~h}$ at room temperature. Immunodetection was carried out using the enhanced chemiluminescence (ECL) Western blotting detection reagent (Amersham Biosciences, Piscataway, NJ, USA).

\section{Quantitative real-time reverse transcription pol-} ymerase chain reaction (RT-PCR)

Total RNA was isolated from cells using Trizol reagent (Molecular Research Center Inc., Cincinnati, OH, USA). RNA concentrations were measured from the absorbance at 260 and $280 \mathrm{~nm}$. Complementary DNA (cDNA) synthesis was performed using a Maxime RT PreMix Kit (Intron Biotechnology, Seoul, South Korea), in accordance with the manufacturer's protocol. The following primers were used for real-time RT-PCR analyses: IL-6, 5'-GTA CTC CAG AAG ACC AGA GG-3' and 5'-TGC TGG TGA CAA CCA CGG CC-3'; IL-1 $\beta$, 5'-CAG GAT GAC ATG AGC ACC-3' and 5'-CTC TGC AGA CTC AAA CTC CAC-3'; TNF- $\alpha, 5^{\prime}$-TTG ACC TCA GCG CTG AGT TG-3' and 5'CCT GTA GCC CAC GTC GTA GC-3'; and $\beta$-actin, 5'-
AGG CTG TGC TGT CCC TGT AT-3' and 5'-ACC CAA GAA GGA AGG CTG GA-3'. The levels of IL-1 $\beta$, IL-6, TNF- $\alpha$, and $\beta$-actin mRNAs were measured using a Chromo 4TM Real-Time PCR Detector (Bio-Rad Laboratories Inc., Hercules, CA, USA). For relative quantification, the reactions were performed using $2 \times$ iQTM SYBR Green Supermix (Bio-Rad). The PCR amplification was performed for 44 cycles, each lasting $20 \mathrm{~s}$ at $95^{\circ} \mathrm{C}$ and $65^{\circ} \mathrm{C}$, and $30 \mathrm{~s}$ at $72^{\circ} \mathrm{C}$, with initial denaturation at $95^{\circ} \mathrm{C}$ for 3 min. Cytokine mRNA levels were compared after correction using the $\beta$-actin mRNA level as an internal standard. Expression level was analyzed by gene expression analysis using the Chromo 4 instrument (Bio-Rad Laboratories Inc., Hercules, CA, USA).

\section{Glucose uptake}

Glucose uptake was detected using fluorescent 2-[N-(7nitrobenz-2-oxa-1,3-diazol-4-yl) amino]-2-deoxyglucose (2NBDG; Invitrogen, Carlsbad, CA, USA). Briefly, hypertrophic 3T3-L1 adipocytes grown in 12-well plates were washed twice with serum-free DMEM and incubated in DMEM containing $0.2 \%$ bovine serum albumin for $4 \mathrm{~h}$. Then, RAW 264.7 cells were added to 3T3-L1 adipocytes and incubated for a further $24 \mathrm{~h}$ before treatment with tangeretin. After $24 \mathrm{~h}$, the cells were washed with KrebsRinger-Hepes (KRH) buffer (Boston BioProducts, Inc., Ashland, MA, USA) and treated with tangeretin $(100 \mu \mathrm{M})$, insulin $(100 \mathrm{nM})$, and 2-NBDG $(100 \mu \mathrm{M})$ for $15 \mathrm{~min}$ at room temperature in dark condition. Glucose uptake was terminated by washing the cells three times with cold PBS. The cells were then lysed and harvested using 1\% Triton $\mathrm{X}-100$. The fluorescence intensity was measured at an excitation of $485 \mathrm{~nm}$ and emission of $520 \mathrm{~nm}$ using a fluorescence micro-reader (Fluostat Optima; BMG Labtech, Germany).

\section{Statistical analysis}


All data are representative of at least three independent experiments. Results are expressed as means \pm standard deviation (SD). Statistical analyses were conducted using SPSS 12.0 (SPSS Inc., Chicago, IL, USA).

\section{RESULTS AND DISCUSSION}

To investigate the effect of tangeretin on inflammatory changes in cocultured hypertrophic adipocytes and macrophages, we first evaluated the cytotoxicity of tangeretin in this system using MTT and LDH assays. Tangeretin did not affect the cytotoxicity up to a concentration of $100 \mu \mathrm{M}$ (Fig. 1A). When the hypertrophic 3T3-L1 cells were cocultured with RAW 264.7 cells for $24 \mathrm{~h}$ and incubated for another $24 \mathrm{~h}$, inflammatory mediators were markedly increased. However, tangeretin treatment significantly decreased NO production and the expression of IL- 6 , IL- $1 \beta$, TNF- $\alpha$ mRNA, and iNOS and COX-2 proteins in a dosedependent manner (Fig. 1B, C, D). These results indicate that tangeretin attenuates the inflammatory response caused by interaction between hypertrophied adipocytes and macrophages. Pro-inflammatory cytokines, including IL-6, IL-1 $\beta$, and TNF- $\alpha$, have been reported to have direct effects on adipose tissue cellular metabolism and insulin resistance (Luca and Olefsky 2008; Suganami et al., 2005; Wang et al. 2008). These anti-inflammatory effects of tangeretin in cocultured hypertrophic adipocytes and macrophages might improve insulin resistance in obese adipose tissue.

Therefore, we investigated the effects of tangeretin on glucose uptake and the insulin signaling pathway in cocultured hypertrophic adipocytes and macrophages. When the hypertrophic adipocytes were cocultured with macrophages, glucose uptake was decreased compared with that in culture without macrophages, indicating that insulin resistance was induced in the coculture of hypertrophic adipocytes and macrophages (Fig. 2A). Tangeretin treatment significantly improved glucose uptake, and co-treatment with tangeretin and insulin further enhanced glucose uptake, in the coculture of hypertrophic adipocytes and macrophages (Fig. 2A). In typical insulin signaling, the binding of insulin to its receptor induces insulin receptor substrate (IRS) phosphorylation, initiating insulin signal transduction. The phosphorylation of IRS activates the phosphatidylinositol-3-kinase (PI3K)/Akt pathway, which in turn stimulates the uptake of glucose into cells by inducing translocation of glucose transporters to the plasma membrane (Kanzaki, 2006). Tangeretin did not affect the phosphorylation of either IRS or Akt (Fig. 2B), suggesting that the effect of tangeretin on glucose uptake might be related to insulin sensitivity.

Several signaling cascades have been shown to be involved in glucose uptake in adipocytes. It has been reported that tangeretin increased glucose uptake via the PI3$\mathrm{K} / \mathrm{Akt}$ and PKA/CREB pathways in murine adipocytes (Onda et al., 2013). Kang et al. (2012) reported that dietary supplementation of immature Citrus sunki peel extracts, which contain high levels of PMFs, including tangeretin, increased the phosphorylation of AMP-activated protein kinase (AMPK) in epididymal adipose tissue in high-fatdiet-induced obese mice. Several phytochemicals improve insulin-independent glucose uptake by stimulating the activity of skeletal muscle AMPK (Kim et al., 2012; Zygmunt et al., 2010). The activation of AMPK inhibits the synthesis of pro-inflammatory cytokines, such as IL-6, IL$1 \beta$, and TNF- $\alpha$, in macrophages (Bijland et al., 2013). Thus, the effect of tangeretin on glucose uptake in cocultured hypertrophic adipocytes and macrophages might be partly exerted via an insulin-independent AMPK pathway, although further study is required to uncover the precise molecular action of tangeretin. In summary, this study suggests that tangeretin exerts beneficial effects by attenuating inflammatory changes and increasing glucose uptake in obese adipose tissue. 
A

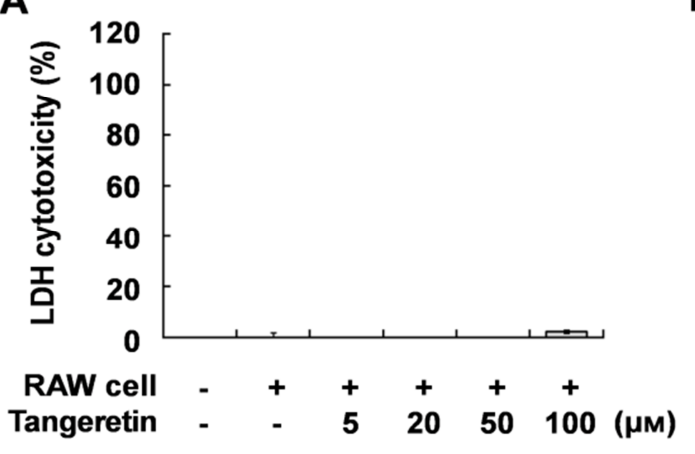

B

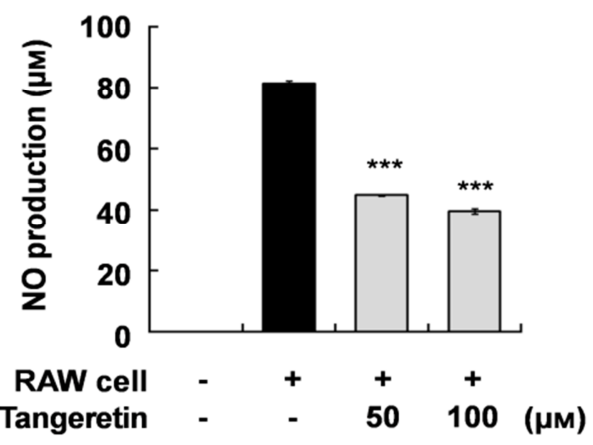

C
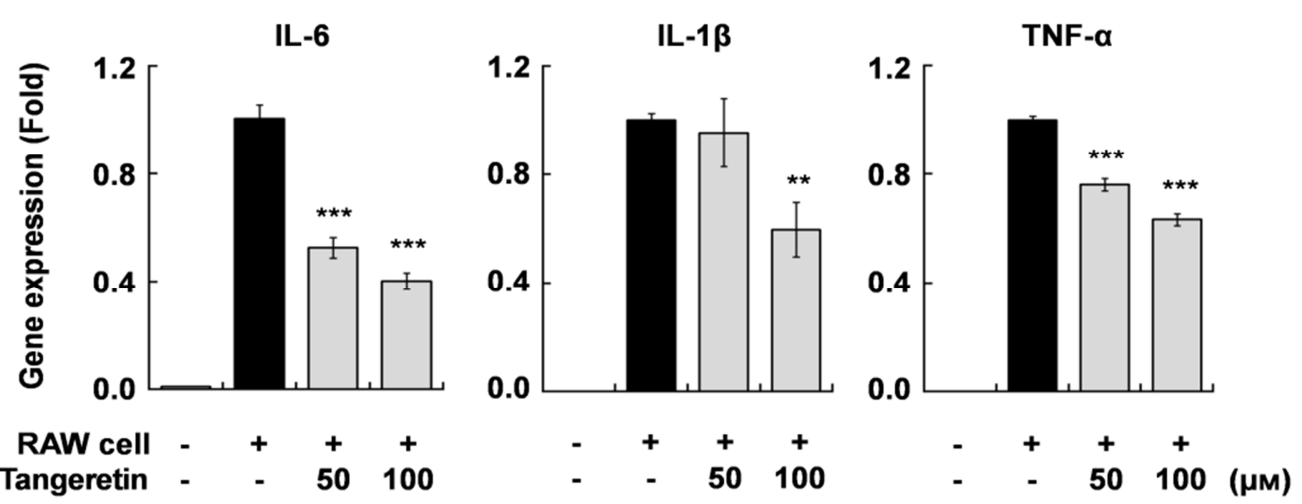

D
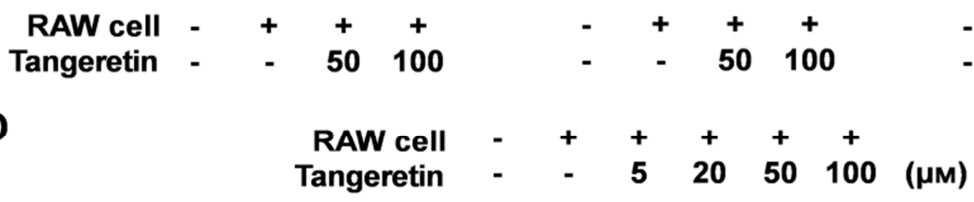

RAW cell $\quad-\quad+\quad+\quad+\quad+$

$\begin{array}{llllllll}\text { Tangeretin } & - & - & 5 & 20 & 50 & 100 & (\mu \mathrm{M})\end{array}$
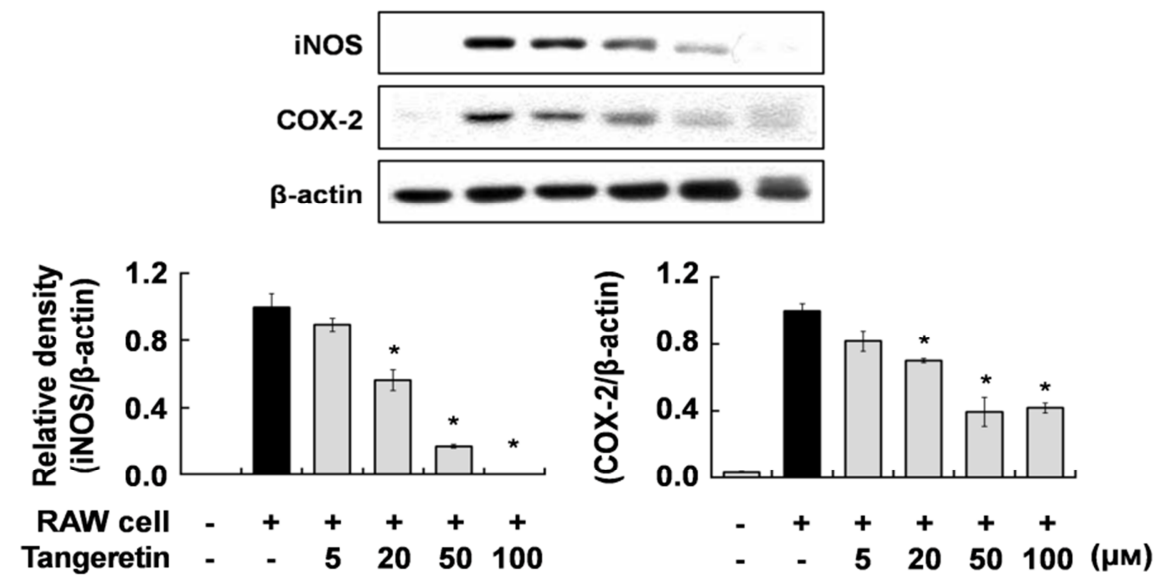

Fig. 1. Effects of tangeretin on the production of inflammatory mediators in cocultured hypertrophic adipocytes and macrophages. RAW 264.7 cells were plated onto serum-starved hypertrophic 3T3-L1 cells and further incubated for $24 \mathrm{~h}$. Then, the cells were treated with the indicated concentrations of tangeretin for $24 \mathrm{~h}$. Lactate dehydrogenase (LDH) cytotoxicity (A), Nitrite production (B), and mRNA levels of cytokines (C) are shown. Induced nitric oxide synthase (iNOS) and COX-2 protein levels (D) were analyzed using Western blot. Relative band density was determined using a densitometer. Target protein expression was normalized to the $\beta$-actin expression level. Data are expressed as means $\pm \mathrm{SD}$ of three determinations $(* p<0.05$, $* * p<0.01$, and $* * * p<0.001$ compared with RAW cells alone). 
A
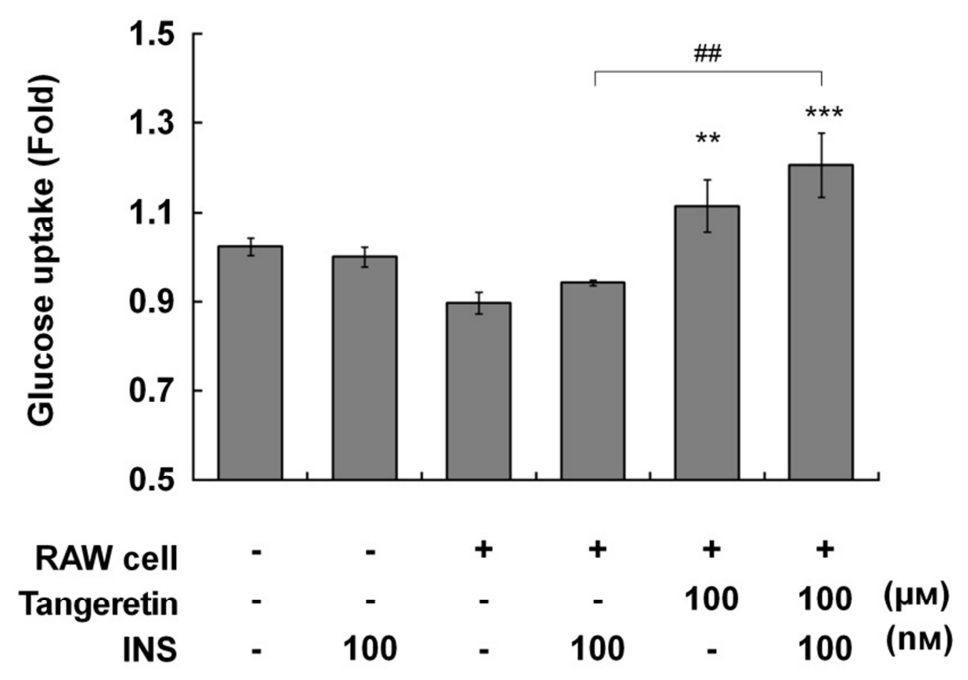

B
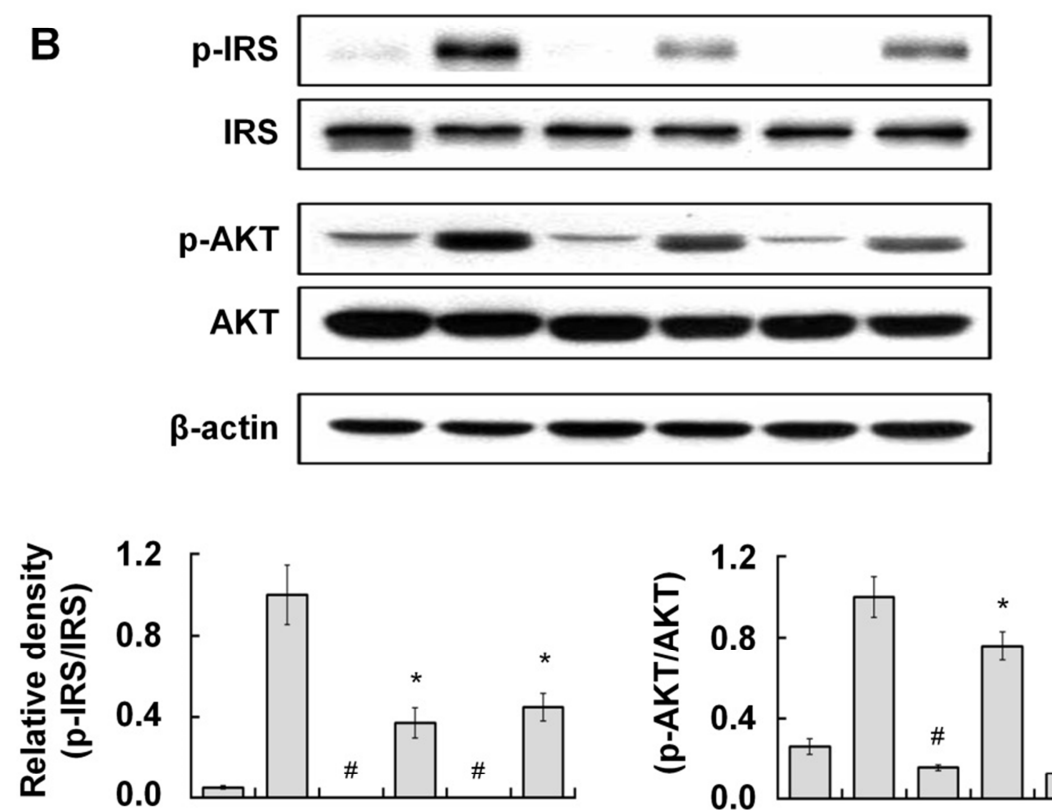

$\begin{array}{rcccccc}\text { RAW cell } & - & - & + & + & + & + \\ \text { Tangeretin } & - & - & - & - & 100 & 100 \\ \text { INS } & - & 100 & - & 100 & - & 100\end{array}$

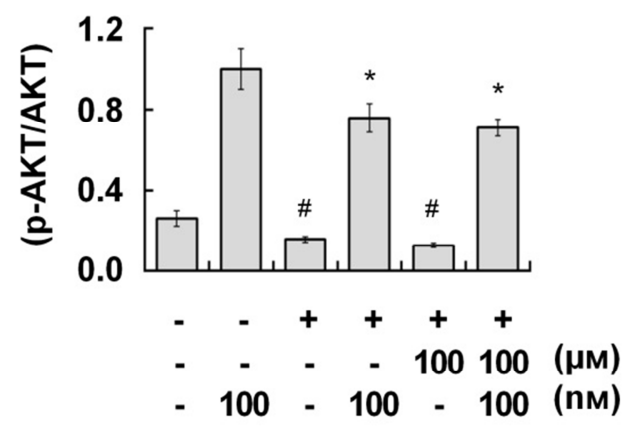

Fig. 2. Effects of tangeretin on glucose uptake and the phosphorylation of insulin receptor substrate (IRS) and Akt in cocultured hypertrophic adipocytes and macrophages. RAW 264.7 cells were plated onto serum-starved hypertrophic 3T3-L1 cells and further incubated for $24 \mathrm{~h}$. Then, cells were incubated with tangeretin $(100 \mu \mathrm{M})$ for 24 h. (A) Glucose uptake was measured in the absence or presence of insulin (100 nM) and 2-[N-(7-nitrobenz-2oxa-1,3-diazol-4-yl) amino]-2-deoxyglucose (2-NBDG) $(50 \mu \mathrm{M})$ and expressed as fold values relative to insulin alone. Data are shown as means $\pm \mathrm{SD}(\mathrm{n}=3 ; * p<0.05, * * p<0.01$, and $* * * p<0.001$ compared with insulin alone; $\# p<0.05$, \#\# $p<0.01$, and \#\#\# $p<0.001$ compared between two groups). (B) Protein levels and their relative band densities were analyzed using Western blot and a densitometer. Data are expressed as means $\pm \mathrm{SD}(* p<0.05$ compared with insulin alone; $\# p<0.05$ compared with negative control). 


\section{ACKNOWLEDGEMENTS}

This research was supported by the 2016 scientific promotion program funded by Jeju National University.

\section{REFERENGES}

Bijland S, Mancini SJ, Salt IP (2013) Role of AMP-activated protein kinase in adipose tissue metabolism and inflammation. Clin Sci 124:491-507.

Chen KH, Weng MS, Lin JK (2007) Tangeretin suppresses IL-1beta-induced cyclooxygenase (COX)-2 expression through inhibition of p38 MAPK, JNK, and AKT activation in human lung carcinoma cells. Biochem Pharmacol 73:215-227.

Choi SY, Ko HC, Ko SY, Hwang JH, Park JG, Kang SH, Han SH, Yun SH, Kim SJ (2007) Correlation between flavonoid content and the NO production inhibitory activity of peel extract from various citrus fruits. Biol Pharm Bull 30:772-778.

Dandona P, Aljada A, Bandyopadhyay A (2004) Inflammation: the link between insulin resistance, obesity and diabetes. Trends Immunol 25:4-7.

Fernández-Real JM, Ricart W (2003) Insulin resistance and chronic cardiovascular inflammatory syndrome. Endocr Rev 24:278-301.

Hotamisligil GS, Arner P, Caro JF, Atkinson RL, Spiegelman BM (1995) Increased adipose tissue expression of tumor necrosis factor-alpha in human obesity and insulin resistance. J Clin Invest 95:2409-2415.

Itoh M, Suganami T, Hachiya R, Ogawa Y (2011) Adipose tissue remodeling as homeostatic inflammation. Int $\mathrm{J}$ Inflam 2011, 720926.

Jeon Y, Lee M, Cheon Y-P (2015). A testa extract of balck soybean (Glycine mac (L.) Merr.) suppresses adipogenic activity of adipose-derived stem cells. Dev Reprod 19:235-242.

Kahn BB, Flier JS (2000) Obesity and insulin resistance. J
Clin Invest 106:473-481.

Kang SI, Shin HS, Kim HM, Hong YS, Yoon SA, Kang SW, Kim JH, Kim MH, Ko HC, Kim SJ (2012) Immature Citrus sunki peel extract exhibit antiobesity effects by $\beta$-oxidation and lipolysis in high-fat diet-induced obese mice. Biol Pharm Bull 35:223-316.

Kanzaki M (2006). Insulin receptor signals regulating GLUT4 translocation and actin dynamics. Endocr J 53:267-293.

Kim MS, Hur HJ, Kwon DY, Hwang JT (2012) Tangeretin stimulates glucose uptake via regulation of AMPK signaling pathways in $\mathrm{C} 2 \mathrm{C} 12$ myotubes and improves glucose tolerance in high-fat diet-induced obese mice. Mol Cell Endocrinol 58:127-234.

Luca CD, Olefsky JM (2008) Inflammation and insulin resistance. FEBS Lett 582:97-105.

Morley KL, Ferguson PJ, Koropatnick J (2007) Tangeretin and nobiletin induce G1 cell cycle arrest but not apoptosis in human breast and colon cancer cells. Cancer Lett 251:168-178.

Nonogaki K, Fuller GM, Fuentes NL, Moser AH, Staprans I, Grunfeld C, Feingold KR (1995) Interleukin-6 stimulates hepatic triglyceride secretion in rats. Endocrinology 136:2143-2149.

Onda K, Horike N, Suzuki T, Hirano T (2013) Polymethoxyflavonoids tangeretin and nobiletin increase glucose uptake in murine adipocytes. Phytother Res 27: 312-316.

Perreault M, Marette A (2001) Targeted disruption of inducible nitric oxide synthase protects against obesitylinked insulin resistance in muscle. Nat Med 7:11381143.

Shin HS, Kang SI, Park DB, Kim SJ (2016) Resveratrol suppresses inflammatory responses and improves glucose uptake in adipocytes interacted with macrophages. Genes Genom 38:137-143.

Spiegelman BM, Flier JS (1996) Adipogenesis and obesity: rounding out the big picture. Cell 87:377-389. 
Suganami T, Nishida J, Ogawa Y (2005) A paracrine loop between adipocytes and macrophages aggravates inflammatory changes: role of free fatty acids and tumor necrosis factor alpha. Arterioscler Thromb Vasc Biol 25:2062-2068.

Sundaram R, Shanthi P, Sachdanandam P (2014) Effect of tangeretin, a polymethoxylated flavone on glucose metabolism in streptozotocin-induced diabetic rats. Phytomedicine 21:793-799.

Wang P, Mariman E, Renes J, Keijer J (2008) The secretory function of adipocytes in the physiology of white adipose tissue. J Cell Physiol 216:3-13.

Weisberg SP, McCann D, Desai M, Rosenbaum M, Leibel RL, Ferrante AW (2003) Obesity is associated with macrophage accumulation in adipose tissue. J Clin Invest 112:1796-1808.
Xu H, Barnes GT, Yang Q, Tan G, Yang D, Chou CJ, Sole J, Nichols A, Ross JS, Tartaglia LA, Chen H (2003) Chronic inflammation in fat plays a crucial role in the development of obesity-related insulin resistance. J Clin Invest 112:1821-1830.

Zhang HH, Halbleib M, Ahmad F, Manganiello VC, Greenberg AS (2002) Tumor necrosis factor-alpha stimulates lipolysis in differentiated human adipocytes through activation of extracellular signal-related kinase and elevation of intracellular cAMP. Diabetes 51:29292935.

Zygmunt K, Faubert B, MacNeil J, Tsiani E (2010) Naringenin, a citrus flavonoid, increases muscle cell glucose uptake via AMPK. Biochem Biophys Res Commun 398:178-83. 